\title{
TRAUMA NO IDOSO
}

\author{
TRAUMA INELDERLY
}

Elcio S Hirano ${ }^{1}$, Gustavo Pereira Fraga² ${ }^{2}$ Mario Mantovani ${ }^{3}$

\begin{abstract}
${ }^{1}$ Médico Assistente. ${ }^{2}$ Docente, Pós-Doutorado na Universidade da Califórnia, San Diego, com bolsa FAPESP. ${ }^{3}$ Docente. Disciplina de Cirurgia do Trauma. Departamento de Cirurgia. Faculdade de Ciências Médicas - UNICAMP.

Correspondência: Elcio S. Hirano. Av. Governador Pedro de Toledo, 638 ap. 91-3 - Bonfim. 13070-752 Campinas - SP. e-mail: elcio@hc.unicamp.br
\end{abstract}

Hirano ES, Fraga GP, Mantovani M. Trauma no idoso. Medicina (Ribeirão Preto) 2007; 40 (3): 352-7, jul./set.

RESUMO: A ocorrência de trauma no idoso vem crescendo de forma significativa, mediante ao crescimento desta população. Atualmente, o estilo de vida mais ativa do idoso eleva a exposição ao risco de acidentes. Além desse fator, o próprio envelhecimento fisiológico contribui: diminuição da acuidade visual, diminuição da audição, uso de medicações, doenças associadas e marcha lentificada. Outros sistemas orgânicos também possuem diminuição de suas funções: renal, pulmonar, cardiovascular, osteomuscular e endócrino. A queda é o mecanismo de lesão mais freqüente, seguido por atropelamento. Após o trauma o idoso possui uma decadência na sua qualidade de vida, não conseguindo retornar plenamente ao seu estado inicial. A prevenção por meio da redução de exposição aos riscos de trauma é a melhor ferramenta para diminuir a morbidade e mortalidade. Qualquer mínima alteração dos parâmetros fisiológicos pode ser indício de uma lesão potencialmente letal. Portanto, a abordagem inicial do idoso traumatizado deve ser realizada com maior atenção e rigorosamente monitorizada.

Descritores: Trauma. Idoso. Fatores de Risco. Assistência Geriatrica.

\section{1- INTRODUÇÃO}

A Organização Mundial da Saúde define como idoso o indivíduo com mais de 65 anos de idade em países desenvolvidos e 60 anos para os países em desenvolvimento ${ }^{1}$.

Atualmente o crescimento da população de idosos é um fenômeno mundial. Em 1950 eram cerca de 204 milhões, em 1998 eram 579 milhões. A projeção para 2050 será de 1,9 bilhão, o equivalente à população infantil de zero a 14 anos. No Brasil, segundo o Censo de 2000, a população de idosos em 1991 era de 10.722.705, e em 2000 era de 14.536.029. As mulheres em 2000 correspondiam a 55,1\% dessa população ${ }^{1}$.

Nos Estados Unidos da América (EUA) o idoso é hospitalizado duas vezes mais que a população restante e a taxa de mortalidade é seis vezes maior do que a do jovem, mediante a comparação realizada em indivíduos com gravidade de lesão semelhante ${ }^{2}$.

O grupo de pacientes idosos vítimas de trauma apresenta uma alta taxa de mortalidade, além de acarretar um gasto elevado de recursos financeiros e um importante custo social ${ }^{3}$. Um outro aspecto relevante no traumatizado idoso é o procedimento anestésico ${ }^{4}$.

Os mecanismos mais freqüentes de trauma são queda e acidentes automobilísticos (atropelamento e colisão), seguidos por queimaduras, violência doméstica e assaltos. Em torno de um terço da população de idosos nos EUA são vítimas de queda por ano, provocando lesões que necessitam de tratamento, e há um aumento para $50 \%$ em idosos com mais de 80 anos $^{5}$. O acidente automobilístico em que o idoso está envolvido geralmente ocorre em baixa velocidade, sem uso de bebida alcoólica ou drogas, e próximo de sua residência. 
Outro aspecto que recentemente tem levantado preocupação nos EUA é a tentativa de suicídio em idosos. Crandall et al. ${ }^{6}$ estudaram 1812 internações por tentativa de suicídio em idosos, o que correspondeu a $9,5 \%$ do total de internações psiquiátricas no período entre 1995 e 2002. Nesse estudo o mecanismo mais utilizado foi arma de fogo. Na Alemanha, Kuhne et al. ${ }^{7}$ realizaram um estudo com 5375 vítimas de trauma com idade maior de 15 anos, no período entre 1993 e 2000 , e observaram uma taxa média de suicídio de $6,4 \%$, e quando se avaliou nas faixas etárias de 15 a 55 anos, de 56 a 75 anos e de 76 a 95 anos, as taxas foram, respectivamente, $9,6 \%, 5,2 \%$ e $6,0 \%$.

Fator importante inerente ao idoso é a recidiva do mesmo trauma, sendo a presença de comorbidades associada a esta recorrência ${ }^{8}$. Deve-se ter muita atenção com a ocorrência de lesão que desvie a atenção do médico e que condicione deixar passar uma lesão despercebida.

\section{2- ENVELHECIMENTO: ALTERAÇÕES FI- SIOLÓGICAS}

A idade cronológica pode não corresponder com a idade fisiológica, isto é, comparando dois pacientes idosos com a mesma idade, um que não possui patologia e o outro que é portador de diabetes mellitus e doença obstrutiva pulmonar crônica, com lesões idênticas, os mesmos apresentam evoluções pós-trauma distintas $^{9}$. Entretanto, essa diferença de resposta ao trauma é observada com menor freqüência na população jovem, pois são poucos indivíduos que possuem patologias sistêmicas, promovendo uma resposta ao trauma mais homogênea nesse grupo.

Evidências clínicas demonstram a diminuição da função fisiológica dos sistemas do corpo humano mediante ao envelhecimento, como: menor complacência pulmonar, diminuição da função renal, menor índice cardíaco e resposta adversa à perda sangüínea.

Após o atendimento inicial e do tratamento definitivo, seja esse cirúrgico ou não, a equipe deve ter maior atenção às complicações clínicas, como por exemplo: isquemia miocárdica, pneumonias, trombose venosa profunda, tromboembolismo pulmonar, tétano e outras.

O envelhecimento promove alterações em todos os sistemas do corpo humano. Dentro do contexto do trauma alguns sistemas são importantes na avaliação inicial e prevenção de possíveis complicações, em particular: renal, respiratório, cardiovascular e osteomuscular.

\section{1- Alterações renais}

A função renal pode se apresentar normal com $90 \%$ de perda do número de néfrons e diminuição de até $50 \%$ do fluxo renal. Esses fatores promovem a diminuição da taxa de filtração glomerular. A diminuição da taxa de filtração glomerular não causa alteração significativa da dosagem da creatinina plasmática. A melhor avaliação é realizada através da determinação do clearance de creatinina, com coleta simultânea de amostra de urina e sangue, podendo utilizar a seguinte fórmula ${ }^{8,10}$ :

Clearence de creatinina $(\mathrm{mL} / \mathrm{min})=\frac{(140-\text { idade }) \times \text { peso }(\mathrm{kg})}{72 \times \text { creatinina sérica }(\mathrm{mg} / \mathrm{dL})}$
$(15 \%$ menor em mulher $)$

Os valores de referências do clearence de creatinina são:

a) homem: 94 a $140 \mathrm{ml} / \mathrm{min}$

b) mulher: 72 a $110 \mathrm{ml} / \mathrm{min}$

Os néfrons apresentam uma diminuição na sensibilidade ao hormônio anti-diurético, o que pode ocasionar um estado de desidratação crônica. $\mathrm{O}$ uso de antibióticos devem ter sua dosagem calculada conforme a função renal. ${ }^{2} \mathrm{O}$ uso de aminoglicosídeos deve ser cauteloso devido a nefrotoxidade, e quando possível substituí-los por outra categoria com o mesmo espectro de ação.

\section{2- Alterações respiratórias}

Ocorre diminuição do comprimento e aumento da distância antero-posterior da caixa torácica, com sobrecarga da função diafragmática. A estrutura óssea torna-se mais vulnerável às fraturas. No parênquima pulmonar ocorre diminuição da elastina, do volume e área alveolar, e diminuição da função mucociliar. As camadas íntima e média das artérias pulmonares se espessam causando elevação da resistência vascular pulmonar ${ }^{8,11}$. Essas alterações fisiológicas promovem um aumento do trabalho respiratório, conseqüentemente sobrecarregando o sistema cardiovascular, além do risco maior de infecção pulmonar.

A flora da orofaringe e das vias aéreas superiores possuem predomínio de bactérias gram-negativas devido à diminuição do tônus do esfíncter esofagiano inferior. Associado ao menor clearance do sistema mucociliar há maior incidência de complicações infecciosas e de broncoaspiração ${ }^{10}$. 


\section{3- Alterações cardiovasculares}

As complicações cardiovasculares são as mais prevalentes e a principal causa de óbito no idoso traumatizado ${ }^{10}$.

O sistema cardiovascular é que se manifesta primeiramente de modo inadequado mediante ao trauma devido às alterações do envelhecimento.

O sistema condutor do miocárdio e os receptores beta-adrenérgicos apresentam diminuição de sua resposta às demandas do sistema cardiovascular, fazendo com que ocorra uma menor resposta na freqüência cardíaca e na contratilidade cardíaca.

As alterações são favorecidas pela doença aterosclerótica progressiva, que limita a efetividade da resposta do miocárdio ao estresse por causa da calcificação e perda da elasticidade das artérias coronárias e grandes vasos. Essa situação pode provocar isquemia miocárdica nas regiões distais às obstruções ${ }^{8,10}$.

Essas alterações fisiológicas podem interferir na avaliação inicial, condicionando que a reanimação volêmica possa ser com sobrecarga ou insuficiente. Portanto, a melhor avaliação do sistema cardiovascular é a monitorização invasiva com um cateter na artéria pulmonar, que também orienta o manejo de drogas vasoativas ${ }^{10}$.

\section{4- Alterações osteomusculares}

A mobilidade e a velocidade dos movimentos diminuem devido ao enrigecimento dos ligamentos $\mathrm{e}$ da degeneração das cartilagens ${ }^{10}$.

Ocorre diminuição da massa óssea devido à osteosporose, propiciando a ocorrência de fratura com mínimo trauma. Há também diminuição da massa muscular e aumento do tecido adiposo, diminuindo a força muscular e a necessidade calórica diáriaa ${ }^{8,11}$.

Devido às alterações ósseas pode haver dificuldade na interpretação de exames radiográficos no diagnóstico de fraturas. A alta suspeição é necessária, pois $50 \%$ das fraturas por compressão são assintomáticas no idoso ${ }^{10}$. Outra dificuldade é o manejo da via aérea devido à limitação da mobilidade da mandíbula e alterações ósseas da coluna vertebral cervical.

\section{3- DOENÇAS ASSOCIADAS E MORTALIDADE}

Uma situação importante é a presença de doenças associadas que possam desviar a atenção para o diagnóstico de lesões, condicionando que uma lesão seja despercebida. Por exemplo: idoso com seqüela de acidente vascular cerebral no hemicorpo direito encontrado caído no quarto ao lado de sua cama, relata que tentou se levantar sem ajuda, com pequenos hematomas em membros superiores e sem alteração do nível de consciência ou amnésia lacunar. Deve-se avaliar com exames radiográficos, além dos membros superiores, o membro inferior direito, para excluir fratura.

O uso de medicações pode interferir na resposta ao trauma, dificultando a avaliação inicial, como na reanimação, como por exemplo, em usuários de bloqueadores de canais de cálcio e beta-bloqueadores.

A presença de marca-passo mantém uma freqüência constante, que não aumenta conforme a necessidade de um aporte maior de oxigenação dos tecidos mediante a um estresse, ou seja, trauma.

O uso de anticoagulante sistêmico pode ocasionar maiores perdas sangüíneas, aumentando o risco para choque hipovolêmico ou dificultando o tratamento do mesmo. Essa medicação também aumenta o risco de sangramento intracerebral após trauma craniencefálico ${ }^{7}$.

Milzman et al. ${ }^{12}$ identificaram em um estudo de 7798 vítimas adultas um aumento da incidência de doenças associadas conforme o envelhecimento. $\mathrm{Na}$ quarta década de vida há uma prevalência de $17 \%$, na sexta década de vida eleva-se para $44 \%$ e acima de 75 anos atinge $65 \%$ da população. Estes autores demonstraram a associação entre a presença de doenças associadas no adulto (jovem e idoso) traumatizado com a idade e a evolução (Tabela I). Os idosos traumatizados com mais de 60 anos apresentaram maior taxa de mortalidade em comparação aos jovens traumatizados $(9,2 \%$ versus $3,2 \%)$.

\begin{tabular}{l} 
Tabela I: Correlação entre a presença de doenças as- \\
sociadas e a mortalidade no trauma \\
\hline \begin{tabular}{cccr}
\hline $\begin{array}{c}\mathrm{N}^{\circ} \text { de doenças } \\
\text { associadas }\end{array}$ & $\begin{array}{c}\mathrm{N}^{\circ} \text { de } \\
\text { vítimas }\end{array}$ & $\begin{array}{c}\text { Sobreviventes } \\
(\%)\end{array}$ & Mortalidade \\
\hline 0 & 6552 & 6341 & $211(3,2 \%)$ \\
1 & 924 & 868 & $56(6,1 \%)$ \\
2 & 233 & 197 & $36(15,5 \%)$ \\
$\geq 3$ & 89 & 67 & $22(24,9 \%)$ \\
\hline
\end{tabular}
\end{tabular}

Kuhne et al. ${ }^{7}$ no seu estudo, onde dividiram as vítimas em três grupos de faixa etárias (15-55 anos, 56-75 anos e 76-95 anos), demonstraram que ocorre maior taxa de mortalidade entre 56-75 anos de idade, 
independente da gravidade da lesão, se comparado ao grupo de pacientes mais jovens. Nesse estudo a taxa média de mortalidade foi de $11,8 \%$ para o grupo de idosos traumatizados, enquanto no grupo mais jovem foi de $5,1 \%$.

\section{4- GRAVIDADE DAS LESÕES}

Estudos retrospectivos não definem claramente fatores preditivos de gravidade, entretanto a presença de lesões complexas de extremidades, craniencefálica ou raquimedular apresentam alto índice de complicações e mortalidade $8,9,11$.

Os índices de trauma fisiológicos e anatômicos podem demonstrar correlação com o prognóstico: Revised Trauma Score (RTS) ${ }^{13}$, Injury Severity Score (ISS) ${ }^{14}$, Acute Physiology and Chronic Health Evaluation Score (APACHE II) ${ }^{15}$ e Geriatric Trauma Survival Score (GTSS) ${ }^{16}$. Um parâmetro bioquímico que pode ser utilizado no idoso traumatizado para avaliar a reanimação volêmica é a determinação da reserva de base ou base excess ${ }^{9}$. Os índices que utilizam somente parâmetros fisiológicos podem identificar os idosos traumatizados que se beneficiariam com uma reanimação agressiva ${ }^{9}$. O GTSS e APACHE II são índices que utilizam a idade, podendo ter valor preditivo de mortalidade.

O RTS é determinado pelos fatores: pressão arterial sistólica, freqüência respiratória e escala de coma de Glasgow ${ }^{13}$. Esses fatores avaliam três sistemas importantes e críticos para o prognóstico do idoso traumatizado: cardiovascular, respiratório e sistema nervoso central. O RTS determina o real estado fisiológico do traumatizado, seja no atendimento préhospitalar ou na sala de emergência ${ }^{13}$. Entretanto, na presença de uma lesão branda, mediante a menor reserva fisiológica do idoso, esse índice pode se apresentar diminuído.

O ISS é um índice que pode se correlacionar com evolução do idoso traumatizado podendo indicar quais evoluem para complicações pulmonares ou gastrointestinais ${ }^{8,11,14}$. van der Sluis et al. ${ }^{17}$ estudaram 121 vítimas de trauma com 60 anos ou mais, sendo que aqueles que obtiveram valor igual ou maior que 50 não sobreviveram e que esse índice não deve ser utilizado individualmente. Tornetta et al. ${ }^{18}$ demonstraram outros fatores preditores além do ISS, que foram: transfusão de grande volume de hemoderivados, necessidade de reposição volêmica e trauma craniencefálico grave ou moderado. Por outro lado, concluíram que as lesões ortopédicas tratadas precocemente (menos que 24 horas) e o tempo cirúrgico desses procedimentos não apresentam correlação com o aparecimento de complicações ou alteração da taxa de mortalidade.

Sharma et al. ${ }^{19}$ evidenciaram maior taxa de mortalidade no idoso traumatizado comparado com o adulto jovem quando apresentavam ISS $>25$, sendo as taxas de $43,5 \%$ e $23,8 \%$, respectivamente. O grupo de adultos jovens traumatizados que apresentavam ISS $>50$ teve uma sobrevida de $31 \%$. Kuhne et al. ${ }^{7}$ estratificaram os pacientes em três grupos conforme valor do ISS: Grupo I (16 a 24), Grupo II (25 a 50) e Grupo III (51 a 75). Também estratificaram conforme a idade, em grupos de $10 \mathrm{em} 10$ anos, iniciando com a idade de 15 anos. A mortalidade aumenta significativamente a partir dos 56 anos de idade (Tabela II).

Carrillo et al. ${ }^{20}$ demonstraram que o APACHE II correlacionou-se também com a mortalidade, em trauma fechado, sendo que o seu valor maior ou igual a 15, associado com ISS $\geq 30$ demonstra maior acurácia.

O GTSS utiliza o valor do ISS, associado à idade e a presença ou não de complicações infecciosas e cardíaca para estimar o prognóstico da evolução do idoso traumatizado ${ }^{16}$.

A determinação do consumo da reserva de base (RB) dentro de uma hora após a admissão hospitalar foi correlacionada com o tempo de permanência e mortalidade. A magnitude do consumo da RB pode ser leve $(-3 a-5)$, moderada (- 6 a -9$)$ ou grave $(\leq-10)$. Conforme a literatura, idosos traumatizados

Tabela II: Taxa de mortalidade para diferentes faixas etárias no trauma, conforme gravidade da lesão anatômica (ISS) onde se observa aumento da taxa de mortalidade nos pacientes com idade maior ou igual a 56 anos

\begin{tabular}{|c|c|c|c|c|c|c|c|c|}
\hline \multirow[b]{2}{*}{$I S S$} & \multicolumn{3}{|c|}{ Idade (anos) } & \multirow[b]{2}{*}{$46-55$} & \multirow[b]{2}{*}{$56-65$} & \multirow[b]{2}{*}{$66-75$} & \multirow[b]{2}{*}{$76-85$} & \multirow[b]{2}{*}{$86-95$} \\
\hline & $15-25$ & $26-35$ & $36-45$ & & & & & \\
\hline $16-24$ & 3,4 & 3,4 & 5 & 7,3 & 13 & 22,4 & 31 & 42,9 \\
\hline $25-50$ & 22,1 & 19,9 & 23,4 & 23,8 & 32,1 & 45,7 & 63,5 & 58,8 \\
\hline $50-75$ & 61,9 & 56,9 & 66,7 & 62,2 & 82,1 & 90 & 100 & 100 \\
\hline
\end{tabular}


com consumo grave da RB tiveram uma mortalidade de $80 \%$, com consumo moderado de $60 \%$ e naqueles que se apresentavam normal ou com leve alteração da RB a mortalidade foi de $24 \% 7$. Portanto, a determinação da RB nesses pacientes é essencial para detecção precoce de choque hipovolêmico.

\section{5- CONCLUSÕES}

$\mathrm{O}$ atendimento inicial ao idoso traumatizado segue as mesmas prioridades ao do jovem, entretanto mediante à diminuição da reserva fisiológica e da presença ou não de doenças associadas, esse tipo de paciente torna-se um desafio ao cirurgião do trauma pelas respostas adversas que apresenta após reanimação, e/ou procedimento cirúrgico, e/ou internação em unidade de terapia intensiva.
Portanto, o cirurgião do trauma deve ter a preocupação em:

- Realizar procedimentos precoces (exemplo: intubação endotraqueal);

- Tratamento precoce e eficaz da dor;

- Alta suspeição de lesão despercebida;

- Prevenção de hipotermia, hipoglicemia e/ou hiperglicemia;

Monitorização adequada conforme a cinemática do trauma: pressão venosa central, oximetria de pulso, cardioscópio, gasometria arterial (parâmetros metabólicos e dos gases sanguíneos), controle de exames laboratoriais (exemplo: função renal e eletrólitos) e outros.

Hirano ES, Fraga GP, Mantovani M. Trauma in elderly. Medicina (Ribeirão Preto) 2007; 40 (3): 352-7, july/sept.

ABSTRACT: The occurrence of trauma in the elderly is growing significantly due to the increase of number of this population. Currently, the more active lifestyle of the elderly leads them to be more exposed to the risk of accidents. Besides that, physiological ageing itself contributes to such risk: reduction of sight, reduction of hearing, use of medication, associated diseases and slow locomotion. Other organic systems also present reduction of their functions: renal, pulmonary, cardiovascular, musculoskeletal and endocrine. Fall is the most frequent mechanism of trauma, followed by motor vehicle crash. After the trauma there is a decline in the quality of life of the elderly since they have difficulty to fully recover. The best method to diminish morbidity and mortality is to prevent the elderly from being exposed to risks. Minimum alteration of the physiological parameters can suggest potentially lethal injury. Therefore, the initial approach to the traumatized elderly should be with careful attention and rigorous monitoring.

Keywords: Trauma. Elderly. Risk Factors. Geriatric Care.

\section{REFERÊNCIAS}

1 - IBGE. Estudos \& Pesquisas - Informações demográfica e socioeconômica. O fenômeno mundial. Perfil dos idosos responsáveis pelos domicílios no Brasil 2000. Disponível em: www.ibge.gov.br/home/estatistica/populacao/ perfilidosos2000.pdf. Acesso em 12 de julho de 2007.

2 - Schwab CW. Trauma geriátrico. In: Moore EE, Mattox KL, Feliciano DV, editores. Manual do trauma. $4^{\mathrm{a}} \mathrm{ed}$. Porto Alegre: Art Med; 2006. p.476-82.

3 - Chang TT, Schecter WP. Injury in the erderly and end-of-life decisions. Surg Clin North Am 2007; 87(1): 229-45.

4 - Lewis MC, Abouelenin K, Paniagua M. Geriatric trauma: special considerations in the anesthetic management of the injured elderly patient. Anesthesiol Clin 2007; 25(1): 75-90.
5 - Tinetti ME. Performance-oriented assessment of mobility problems in elderly patients. J Am Geriatr Soc 1986; 34(2): 119-26.

6 - Crandall M, Luchette F, Esposito TJ, West M, Shapiro M, Bulger E. Attempted suicide and the elderly trauma patients: risk factors and outcomes. J Trauma 2007; 62(4): 1021-7.

7 - Kuhne CA, Ruchholtz S, Kaiser GM, Nast-Kolv D. Mortality in severely injured elderly trauma patients - When does become a risk factor? World J Surg 2005; 29(11): 1476-82.

8 - McMahon DJ, Shapiro MB, Kauder DR. The injured elderly in the trauma intensive care unit. Surg Clin North Am 2000; 80(3): 1005-19.

9 - Jacobs DG, Plaisier BR, Barie PS, Hammond JS, Holevar MR, Sinclair KE et al. Practice management guidelines for geriatric trauma: the EAST practice management guidelines work group. J Trauma 2003; 54(2): 391-416. 
10 - Schwab CW, Shapiro MB, Kauder DR. Geriatric trauma: patterns, care, and outcomes. In: Mattox KL, Feliciano DV, Moore EE, editors. Trauma. 4th ed. New York: McGraw-Hill; 2000. p.1099-114.

11 - Souza JAG, Iglesias ACRG. Trauma no idoso. Rev Assoc Med Bras 2002; 48(10): 79-86.

12 - Milzman DP, Boulanger BR, Rodrigues A, Soderstrom CA Mitchell KA, Magnant CM. Pré-exiting disease in trauma patients: a predictor of fate independent of age and injury severity score. J Trauma 1992; 32(2): 236-43.

13 - Champion HR, Sacco WJ, Copes WS, Gann TS, Gennarelli TA, Flanagan ME. A revision of the Trauma Score. J Trauma 1989; 29(5): 623-9.

14 - Baker SP, O'Neill B, Haddon W Jr, Long WB: The injury severity score: a method for describing patients with multiple injuries and evaluating emergency care. J Trauma 1974; 14(2): 187-96.

15 - Knaus WA, Draper EA, Wagner DP, Zimmerman JE: APACHE II: A severity of disease classification system. Crit Care Med 1985; 13(10): 818-29.
16 - DeMaria EJ, Kenney PR, Merrian MA, Casanova LA, Gann DS. Survival after trauma in geriatric patients. Ann Surg 1987; 206(6): 738-43.

17 - van der Sluis CK, Klasen HJ, Eisma WH, ten Duis HJ. Major trauma in young and old: what is the difference? J Trauma 1996; 40(1): 78-82.

18 - Tornetta P, Mostafavi H, Riina J, Turen C, Reimer B, Levine R, et al. Morbidity and mortality in elderly trauma patients. $\mathrm{J}$ Trauma 1999; 46(4): 702-6.

19 - Sharma OP, Oswanski MF, Sharma V, Stringfellow K, Raj SS. An apprasial of trauma in the elderly. Am Surg 2007; 73(4): 354-8.

20 - Carillo EH, Richardson JD, Malias MA, Cryer HM, Miller FB. Long term outcome of blunt trauma care in the elderly. Surg Gynecol Obstet 1993; 176(6): 559-64.

Recebido em 26/06/2007

Aprovado em 23/08/2007 